\title{
Papeln an Augenlidern, Lippen und Zunge
}

— Bei einem 16-jährigen Jungen fanden sich multiple Papeln und Knötchen an den Augenlidern, den Lippen und der Zunge (Abb. A, B, C). Sechs Jahre zuvor war bei seiner Mutter im Alter von 38 Jahren die Diagnose eines metastasierenden medullären Schilddrüsenkarzinoms gestellt worden.

Zum damaligen Zeitpunkt waren bei ihren beiden Söhnen deutlich erhöhte Kalzitoninwerte, allerdings kein laborchemischer Hinweis für ein Phäochromozytom, festgestellt

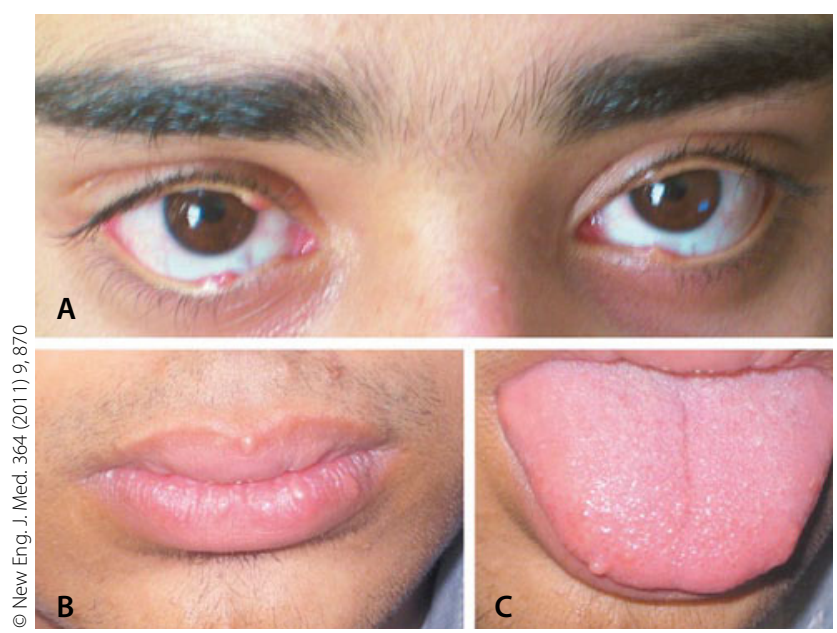

Papeln und Knötchen an Augenlidern, Lippen und Zunge des 16-jährigen Patienten. worden. Bei beiden Jungen führte man daraufhin eine totale Thyreoidektomie durch. Die sorgfältige pathologische Aufarbeitung der Schilddrüse dieses Jungen erbrachte ebenfalls ein medulläres Schilddrüsenkarzinom, allerdings nur in einem Durchmesser von $0,5 \times 0,5 \mathrm{~cm}$.

Die Konstellation und die Anamnese der drei Personen sprechen für das Vorliegen einer multiplen endokrinen Neoplasie (MEN) Typ 2B, einer autosomal-dominant vererbten Erkrankung, die durch ein medulläres Schilddrüsenkarzinom, ein Phäochromozytom, intestinale Ganglioneurinome und einen marfanoiden Habitus gekennzeichnet ist. Zur Sicherung der Diagnose ist heute eine Genotypisierung des RET-Protoonkogens möglich. Zum Zeitpunkt der Erkrankung des Jungen stand diese Methode noch nicht zur Verfügung. Die Genotypisierung ist für die betroffenen Familienmitglieder von großer Bedeutung für die genetische Beratung, ein Screening auf ein Phäochromozytom und die prophylaktische Thyreoidektomie. Der Junge erhält eine Substitution mit L-Thyroxin und unterzieht sich fortlaufend Screeninguntersuchungen.

Die auf den ersten Blick rätselhaften Befunde an Lidern, Lippen und Zunge erhalten einen ganz anderen Kontext, wenn man die Familienanamnese kennt.

H. S. FÜESSL .

\section{- M. R. M. Tehrani, M. Aalaa}

(mrmohajeri@sina.tums.ac.ir): Papules on the Eyelids, Lips, and Tongue. New Eng. J. Med. 364 (2011) 9, 870

\section{Übergewicht erhöht das Demenzrisiko}

- Wissenschaftler des Karolinska Instituts in Stockholm prüften den Zusammenhang von Übergewicht und Fettleibigkeit im mittleren Lebensalter und Demenz (vaskuläre Demenz, Alzheimer und andere Demenzformen) im späteren Leben.

Analysiert wurden die Daten von 8534 Zwillingen des schwedischen Zwillingsregisters (Alter über 65, im Mittel 74,4 Jahre). Bei 350 von ihnen wurde eine Demenz diagnostiziert, für 114 bestand der Verdacht auf eine Demenz. Zusätzlich waren die Daten über Körpergröße und Gewicht vor 30 Jahren verfügbar, sodass der damalige Body Mass Index (BMI) berechnet werden konnte. So konnten die Probanden in vier Gruppen eingeteilt werden: untergewichtig, normalgewichtig, übergewichtig und fettleibig (jeweils im mittleren Lebensalter).

29,8\% der Probanden waren 30 Jahre zuvor übergewichtig (BMI >25-30) oder fettleibig ( BMI >30) gewesen. Übergewicht in diesem Alter erhöhte das Risiko für Demenzerkrankungen im späteren Alter (im Vergleich zu Menschen mit normalem BMI) um 80\% (95\%-KI für Übergewichtige 1,71, für Fettleibige 3,88; $\mathrm{p}=0,019)$. Der Zusammenhang zwischen hohem BMI und Altersdemenz blieb auch bestehen, wenn andere Faktoren, wie Bildungsstand, Diabetes und Gefäßerkrankungen in den Berechnungen berücksichtigt wurden

\section{Kommentar}

Sowohl das Übergewicht als auch die Fettleibigkeit im mittleren Lebensalter erhöhen unabhängig von anderen Faktoren das Risiko, später an einer Demenz zu erkranken. Genetische Umweltfaktoren dürften bei dieser Assoziation eine wesentliche Rolle spielen.

K. MALBerg =

\footnotetext{
- W. L. Xu et al.

Midlife overweight and obesity increase latelife dementia risk: A population-based twin study. Neurology 76 (2011) 1568-1574
} 